\section{Conference Report:} The 18th Santa Fe

\section{Symposium}

Manufacturing

Technology

\author{
Mark Grimwade \\ Consultant to World Gold Council, Northwood, \\ Middlesex, UK
}

The 18th Santa Fe Symposium on Jewellery Manufacturing Technology was held at Albuquerque, New Mexico, USA on 23-26 May 2004. It was attended by about 140 registrants of whom almost one-third came from outside of the USA. Twenty-three presentations were given over three and a half days. In addition, Andrea Hill gave three short introductory talks at the start of the first three days continuing her series on management techniques.

\section{"The Nature of Metals and Alloys" Mark Grimwade, Consultant to the Worshipful Company of Goldsmiths, UK}

This was a repeat of the start of a cycle of presentations on "Introduction of Metallurgy" intended to introduce new attendees to the metallurgical concepts underlying alloying behaviour. These were discussed with particular reference to the carat gold and silver alloys. The paper is to be found in the Proceedings of the 15th Symposium published in 2001.

\section{“Is 'Pure' Gold Really Pure?”}

Dr. Valerio Faccenda and Michele Condò, Pomellato S.p.A., Italy

Dr. Faccenda began by describing the "Standard Specification for Refined Gold" ASTM B - 562 which gives four standards ranging from 995 to 999.95 fineness. It does not give upper limits for impurities in 'good delivery bars' of 995 fineness but it lists many of the impurity elements in the higher standards. These may give rise to serious quality problems in jewellery manufacture and he went on to discuss a number of case studies. For example, hard spots and 'comet tails' can appear on the final polished surfaces of pieces due to the presence of platinum group metals (PGMs) such as osmium, iridium, rhodium and ruthenium and metals such as tungsten and cobalt. Some of these impurities come from refined scrap that may have had iridium and cobalt as grain refiners. He concluded that it is preferable to purchase only 999.9 fineness bars and certified with a process able to remove deleterious PGMs.

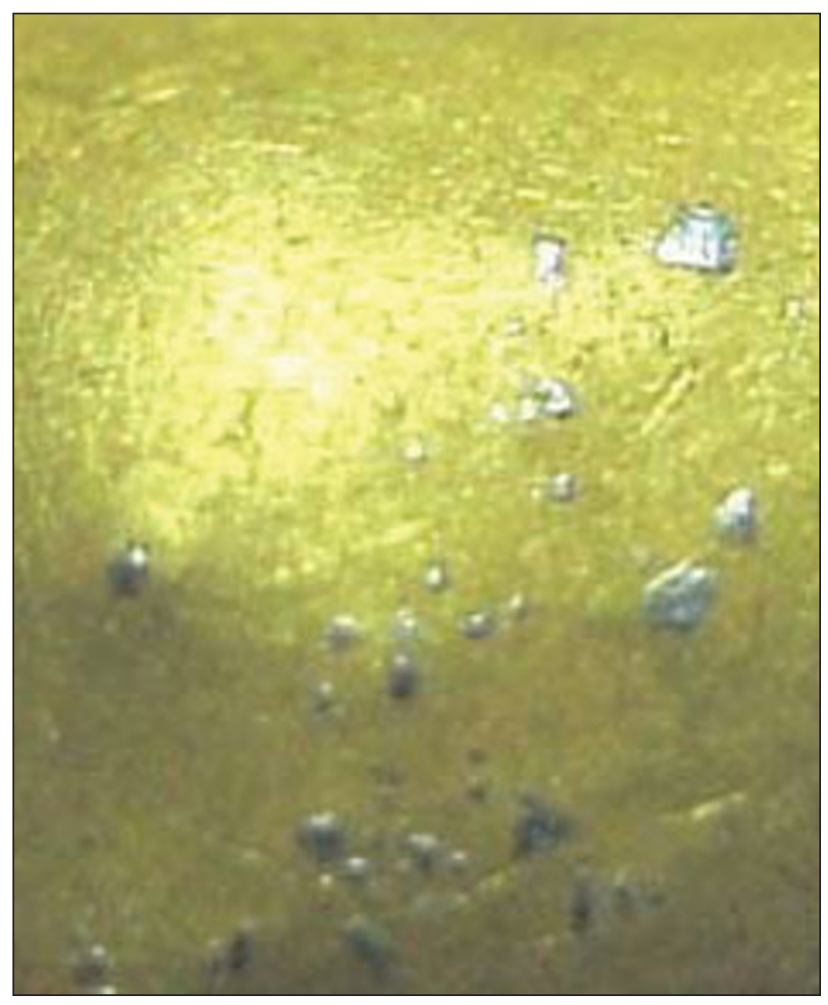

Figure 1

Iridium inclusions in gold bars (V. Faccenda, Pomellato Srl, Italy) 


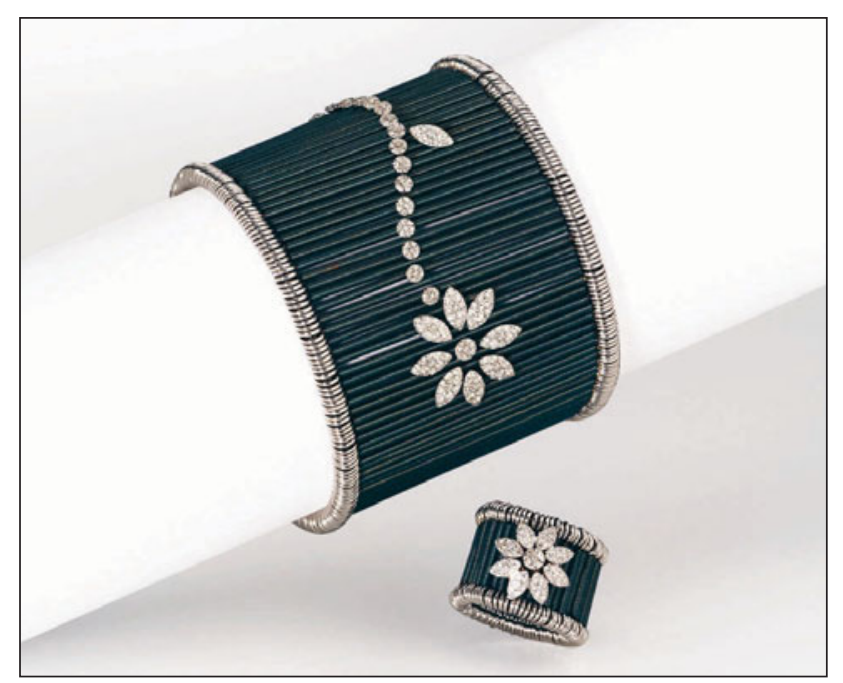

Figure 2

Blue Gold jewellery by Jarretiere, Italy

\section{"Blue, Black and Purple! The Special Colours of Gold"}

Dr. Christopher Corti, World Gold Council, UK

Conventional carat gold jewellery can be produced with colours in different shades of red, yellow, green and white depending on the alloy composition. It is possible also to obtain special colours such as blue, black and purple by alloying gold with other metals to produce intermetallic compounds at fixed compositions. The best known examples are 'purple gold', which is based on the compound $\mathrm{AuAl}_{2}$ at about 79 wt.\% Au - 21 wt.\%Al, and the ice-blue gold-indium compound Auln $\mathrm{n}_{2}$ at 46 wt.\% Au. These compounds are inherently brittle and cannot be worked. Consequently, their uses in jewellery are limited although Dr. Corti discussed some methods for overcoming this problem. Other approaches are to produce coloured coatings onto gold jewellery by electroplating, oxidation, chemical vapour deposition and patination and examples were given of these techniques.

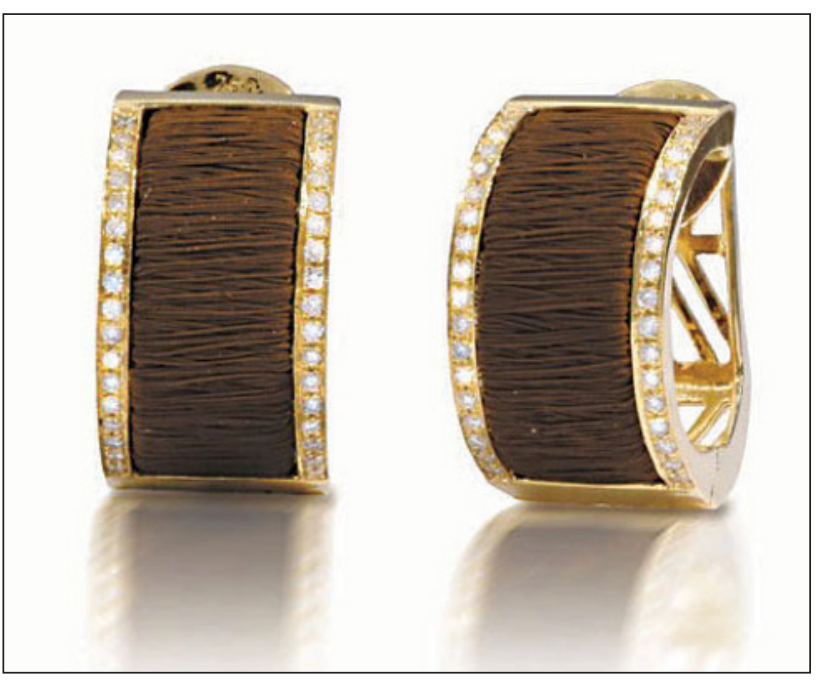

Figure 3

Brown gold jewellery by Yvel, Israel
"Hardening of High Carat Gold Alloys" Dr. Jörg Fischer-Bühner, FEM, Germany

High carat golds such as 22 ct jewellery alloys are comparatively soft in the as-cast and annealed conditions. Increased strength would be desirable for gem setting and for filigree items. This study explored the possibilities for hardening by conventional methods of alloying and heat treatment. The first objective was to establish the most promising additions for increasing as-cast hardness of three 22 ct alloys without impairment of ductility, colour and melting properties. Addition of cobalt (Co) up to $~ 2$ wt.\% proved to be the most effective. Trials on investment castings showed that the hardness increase was dependent on the cooling conditions. Results were given also for $2 \%$ Co alloys after varying age hardening treatments. Pronounced agehardening was achieved with peak hardness levels of 200$260 \mathrm{HV}$, compared with $\sim 60 \mathrm{HV}$ for the standard Au-Ag-Cu alloys. Promising results were obtained also on colour, density, melting range, mechanical properties, corrosion resistance, casting and scrap recycling.

\section{"Reticulation: A Scientific Explanation for the Myth and Technology"}

Stewart Grice, Hoover \& Strong, Inc., USA

Reticulation is the art of producing a rippled and ridged surface texture on alloys. The alloy most commonly used is $80 \%$ silver and 20\% copper although other silver alloys and some carat gold alloys can be reticulated. The technique is to

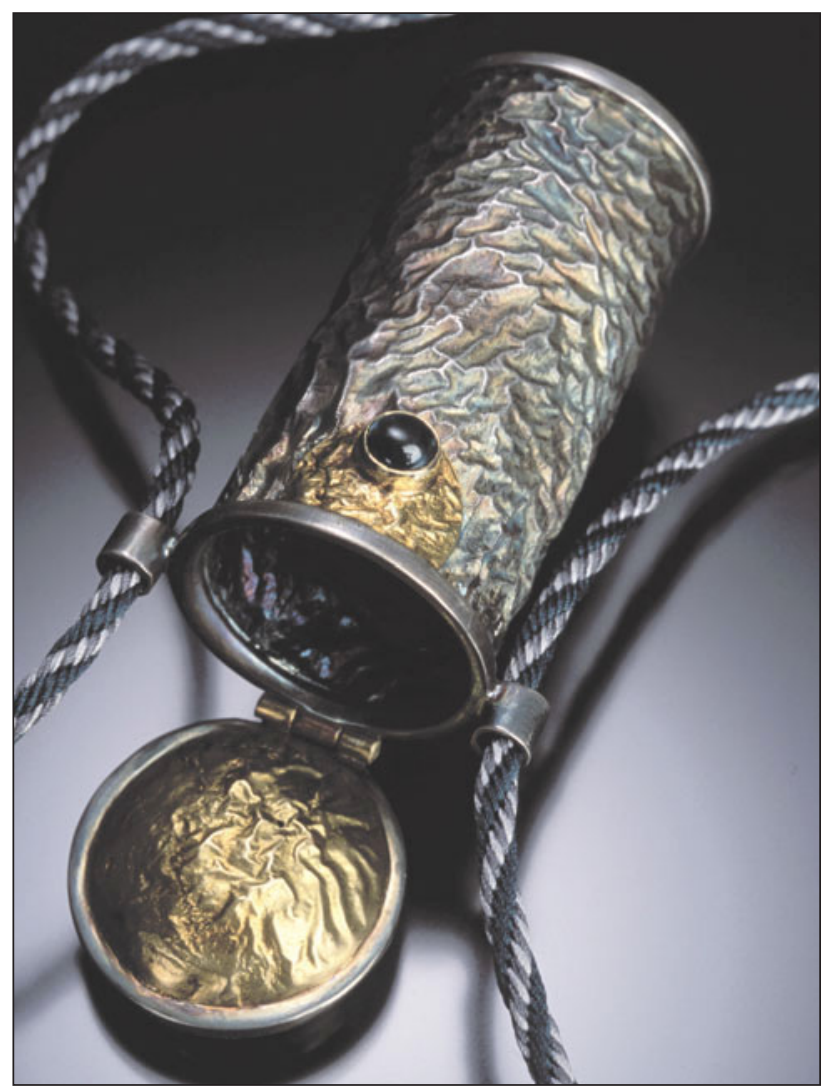

Figure 4

Reticulated 18 carat gold locket by Ann Larsen Hollerbach (photo by R. Diamente, Portland, ME) 
first pre-treat the sheet alloy by multiple oxidation and pickling cycles to remove the copper from the surface layers. This raises the solidus temperature of the surface above the liquidus of the interior. Reticulation is achieved by locally heating the sheet with a suitable torch, gas mix and tip size to melt the interior. The surface texture is generated by expansion and contraction as the interior melts and solidifies and by flame pressure and direction. Stewart Grice described an extensive research programme undertaken to examine all the process variables affecting reticulation. Stunning examples of work by Andrew Nyce and Anne Hollerbach illustrated the technique for jewellery and decorative metalware.

\section{“Non-Traditional Mokumé Gane Materials: Diffusion Bonding of Iron to Precious Metals"}

James Binnion, James Binnion Metal Arts, LLC, USA

Mokumé Gane is a traditional Japanese metalworking technique involving lamination of sheets of copper, silver, gold and their alloys. The laminated blocks are diffusion bonded, forged, incised to different depths and re-worked to produce a patterned surface resembling a wood-grained finish. This can then be patinated to enhance the pattern. The speaker has explored the technique for many years but has more recently been studying combinations of widely differing materials. Here. he described work on laminates of 95\% platinum-5\% palladium to commercially pure iron and a 22 ct yellow gold to iron. Laminates were successfully worked through a wide range of hot and cold reduction processes. The stock could then be used to create finished jewellery.

\section{"Textile Techniques in Metals: A Designer's Perspective on Sheet and Wire"}

\section{Barbara Berk, Barbara Berk Designs, USA}

The presentation was in two parts. In the first, the techniques of weaving and texturing high carat gold and platinum alloy sheet and wire were described. A number of technical problems, such as alloy selection in terms of mechanical strength and 'orange peel' in annealed stock, were met and overcome. The second part reviewed and examined a wide range of pieces made by studio artists and manufacturers.

\section{“Performing 18ct Gold Alloys for Investment Casting"}

Dr. Denis Vincent, Metalor Technologies, Switzerland A major source of defects in investment castings of carat gold alloys are the reactions that produce sulphur dioxide. Dr. Vincent began by discussing the equilibrium between the liquid and gaseous phases in a binary phase diagram. It is possible to get solid and gaseous phases in a non-equilibrium situation particularly when the liquidus is close to the liquid + gas/liquid phase boundary. He went on to discuss the reactions that occur during the decomposition of gypsum (calcium sulphate dihydrate) in terms of its thermodynamic stability in vacuum and in an atmosphere at temperatures experienced in melting and casting. Silicon additions produce a silicon dioxide $\left(\mathrm{SiO}_{2}\right)$ layer between the gypsum and liquid metal preventing liquid metal/investment reactions. However, there are problems of high temperature brittleness and large grain sizes if the addition is too high. (This paper will be published in next year's Proceedings).

\section{"Controlled Flow = Controlled Solidification"}

Apollonius Nooten-Boom II, Hean Studio, UK

This presentation is concerned with methods of controlled flow of molten metal that will eliminate shrinkage porosity in large precious metal castings in the 30-1000 gram range. The speaker explained the problems of producing porosityfree golf putter heads, and other items, in silver, gold and platinum and described a wide variety of sprueing and gating techniques studied in both VPC and centrifugal casting machines. It is not possible to detail all of the work in this extensive study. The main conclusion is that the molten metal should always enter the mould as slowly as possible. For silver and gold, the other variable is mould temperature but with platinum, higher superheat can compensate for cool moulds and slower spin speeds.

\section{"The Role of Surface Area to Volume Ratios and Casting Quality" Tino Volpe, Tiffany \& Co., USA}

An attempt was made in this study to demonstrate the effect of volume-to-surface area (V/A) ratios on casting quality. Four simple geometric shapes were chosen in two sets. One set had the same volume and the other the same surface area. Gating sizes were altered based on Chovorinov's Rule that states that the casting time is proportional to the $(\mathrm{V} / \mathrm{A})^{2}$. Unfortunately, the data were clouded by the fact that the casting temperatures chosen were too high and good castings were not obtained. However, the results did suggest that lowering the temperature might yield good castings. It seemed also that a sprue with a $(\mathrm{V} / \mathrm{A})^{2}$ slightly smaller than that of the casting produced the best quality but more needs to be done in this area.

\section{"Exploring Mould Dilation in Casting jewellery Alloys"} Dr. John Wright, Consultant, UK

Mould dilation is the slight ballooning of the mould cavity and the most important factor is the lack of mould rigidity at the instant of casting. This will affect the dimensions of the casting and it may increase the metal demand beyond the capability of the feeding system leading to interdendritic shrinkage porosity. Although the effect has been seen for other methods of casting, little has been said concerning 
jewellery investment casting. This paper presented evidence of dilation in casting 18ct palladium-white gold and platinum with different investments and casting machines. The main conclusion was that there is a 'safe threshold' of about $4 \%$ dilation below which a good sprueing system should cope with any extra liquid metal feed demand. Dilations as high as $22 \%$ by volume were recorded and it is easily seen at $10 \%$. The 'safe threshold' is dependent on the temperature and kinetic energy of the melt where it hits the mould cavity and on the type and condition of the investment.

\section{“Does Investment Permeability Impact Jewelry Castings?"}

Ralph Carter, Ransom \& Randolph, USA

This paper explored the relationship between gas permeability of the investment and casting fill and surface smoothness for three casting techniques, namely, centrifugal, pressure-over-vacuum and gravity pour with vacuum assist. Permeability constants for mould samples after burnout were measured for different mixed water/powder ratios. Increasing the ratio from $37 / 100$ to $42 / 100$ increased the constant by $37 \%$. The percentage fill of casting grid patterns increased as the permeability constant increased when casting with VPC and gravity pour but was not seen with centrifugal casting. Increasing the constant gave a slight increase in surface roughness of castings.

\section{"Physical-Chemical Analysis of the Factors Influencing the Behaviour of Flasks During Heating in the Jewelry Casting Process: Development of the Optimum Model of a Burnout Furnace" Professor Anatoly Kolomeisky, Rice University, USA and Sergei Kazantsev, Lasso Co., Russia}

Professor Kolomensky reviewed and analysed the important factors influencing the burnout cycle. He began by discussing the advantages and disadvantages of the different types of furnaces from a consideration of the heating method. The chemical processes and reactions taking place in the flask during the heating phase were described. These include removal of the water that saturates the investment, loss of water of crystallisation from the gypsum, melting and burning of the wax and its decomposition products, and reactions involving decomposition of the investment and production of gases at high temperatures. Several directions for technological improvement were outlined.

\section{"Stone-In-Place Casting: The Investment Perspective"}

Dr. Ian McKeer, SRS Ltd., UK

Most stone-in-place casting is done with stones up to $2 \mathrm{~mm}$ in size, as there is an increased risk of thermal shock cracking with larger stones. Stone selection and the problems associated with setting in the wax, wax selection and rubber mould selection were discussed. Stone-casting investments contain additives to protect the stones during burnout and casting. It is not advisable to simply add boric acid to a conventional investment powder, as there is a risk of producing poor quality castings. The recommended burnout cycle for stone-in-place investments must be followed. This includes a maximum temperature of $630^{\circ} \mathrm{C}$, a longer dwell time to ensure good burnout and preferably to break out the casting when the flask is cold. Quenching from high temperature must be avoided.

\section{"Laser Forming as a Method of Producing Designed Objects"}

Dr. Sarah Silve, Brunel University, UK

Laser forming using a $\mathrm{CO}_{2}$ laser is a technique for sheet metal forming in which laser- induced stresses and temperature gradients shape the metal rather than hard tooling and exerted forces. Three bending mechanisms have been identified and these were described. These are a) temperature gradient concave bending, b) buckling (convex bending), and c) upsetting. Processing parameters include the laser energy, laser path feed rate and geometry, the properties of the material being formed, number of scans by the laser beam and the angle of incidence since this affects the absorption of laser energy. A wide variety of shapes utilising the three bending mechanisms illustrated the presentation and these included shapes that are impossible or very difficult to make using conventional tooling. The costs involved in laser forming make it suited for high-value products such as silverware.

\section{"Tack-, Fusion- and Pulse-Arc-Welding Applications for the Bench Jeweler Generalist"}

Mark Mann, Visual Communications, Inc., USA

The processes of tack-, fusion- and pulse-arc welding were described together with typical applications for each. Several advantages are offered by these techniques. For example, it is possible to make a weld on an article of jewellery near a heat-sensitive gemstone without damaging it, jewellery may be assembled from individual components without having to position and torch-solder one at a time, rivets can be welded without freezing the hinge, and repairs can be made to a clasp without annealing it. The paper was well illustrated with examples using carat gold and platinum jewellery.

\section{"Precious Metal Electroplating in the Gold Jewellery Manufacturing Environment"}

Neil Bell, Red Sky Plating, USA

The speaker discussed the equipment and operating procedures required for consistent results when gold and 
rhodium plating onto a carat gold substrate. Emphasis was placed on the importance of good surface preparation prior to plating and in ensuring that safety standards are upheld. Selective plating techniques and the production of special colours, such as black rhodium plating, were described. Finally, some common plating problems along with corrective actions were discussed.

\section{"Applications of the Scanning Electron Microscope for Jewelry Manufacture"} Greg Normandeau, Imperial Smelting \& Refining Co., Canada

The scanning electron microscope (SEM) is an analytical instrument that has many applications for the jewellery industry. This is amply illustrated by the extensive bibliography and list of references given in the Appendix. These include failure analysis, i.e. fractography of broken components, surface examination, and contamination or chemical analysis utilising energy dispersive spectroscopy (EDS). The instrumentation, operating procedures, sample preparation and energy dispersive spectrographic analysis were described in some detail. A number of jewellery related investigations using the SEM were discussed. Few manufacturers can justify the expense of having the SEM and so access is usually on a contract arrangement with a local metallurgical laboratory or university department.

\section{"Micro-Chemical Investigation of Ancient Silver- and Gold-Plated Luxury Objects"} Dr. G. M. Ingo, T. de Caro, M. Marabelli and G. Bultrini, National Research Council of the Institute of Materials Chemistry and the Central Institute for Restoration, Italy

The micro-chemical structures of silver- and gold-plated objects from the Greek, Roman, Barbarian, Dark and Middle Age periods have been studied using different analytical techniques. It should be mentioned that in this context, plating refers not to electroplating but to silver or gold coatings applied by other methods. These include mechanical attachment of thin malleable foil, dipping into molten silver or gold, mercury gilding (fire gilding), oxidation and pickling of copper alloys containing low amounts of precious metal (e.g. depletion gilding) and inverse segregation in copper-silverantimony alloys. Interesting SEM and EDS results were presented for a number of artefacts. In particular, the panels of the golden altar of St. Ambrogio in Milan (829 - 859 AD) were shown to have been fire gilded using a mercury amalgam.

\section{"Judging the Quality of Stone Setting" Arthur Skuratowicz and Julie Nash, Anton Nash LLC, USA}

The aim of this paper was to provide the manufacturing jeweller with a series of quality checks for judging stone settings. The factors affecting the security of stones in various types of settings were explained and amply illustrated. These were metal contact, gaps, metal thickness and quality, cutting of seats, correct stone size, overlapping stones and protruding stones. Visual appeal and short- and long-term hazards were discussed.

\section{“Jewelry and Health: Perspectives for Improvement"}

Andrea Basso, Michele Pertile and Massimo Poliero of Legor Srl, Andrea Peserico and Anna Fortina of University of Padua, Italy

This presentation was concerned with allergies that may occur when items of jewellery come into contact with the skin. The best known of these is the allergic reaction caused by nickel and experienced by at least 10\% of women. Allergic Contact Dermatitis (ACD) from all metals used in jewellery was discussed. Data from experimental metal release studies were presented for a number of 9 and 14 ct gold alloys in the 'as cast', homogenised and mechanically worked conditions. Results were shown to be dependent on condition.

\section{“Industrial Liaison and Training in the UK Jewellery Industry"}

Gay Penfold, Jewellery Industry Innovation Centre, University of Central England, UK

After outlining the history of the Birmingham School of jewellery since its inception in 1889 , the speaker went on to describe the programme of innovative projects and actions taken to improve the dialogue between the jewellery industry and academia. This has led to the formation of the Jewellery Industry Innovation Centre. The Centre conducts research and development and is instrumental in introducing new technologies to the industry, in particular laser welding and rapid prototype modelling. Many companies have used the Centre to conduct feasibility studies on the commercial viability of these and other processes.

\section{“Anti-Money-Laundering Programs Under the USA Patriot Act in the Precious Metals and Jewelry Industry"}

Cecilia Gardner, Jewelers Vigilance Committee, USA

The jewellery industry is highly attractive to money launders and other criminals, including those involved in the funding of terrorism. The rules implementing the anti-moneylaundering requirements of the Patriot Act were discussed.

At the close of the Symposium a number of awards were made. Ambassador Awards were given to Arthur Skuratowicz, Tino Volpe and Martin Moser. The Research Award and Technology Award were presented to Daniele Maggian and Tina Wojtkeilo, respectively. Tyler Teague and Hubert Schuster received Applied Engineering Awards. Jörg FischerBühner received the Collaborative Research Award and Dr. Raj 
Mahesh was given the Award for Outstanding Technical Presentation. An Industry Leader Award was given to Fred Klotz and Mark Grimwade was presented with an Award for Lifetime Achievement.

The Proceedings of this 18th Annual Santa Fe Symposium have been edited by Eddie Bell and published by Met-Chem Research, P.O. Box 67347, Albuquerque, New Mexico 871937347, USA. The book may be obtained from "The Santa Fe Symposium”, 7500 Bluewater Road NW, Albuquerque, New Mexico 87121-1962, USA, Fax: 001505839 3248. Website: www.santafesymposium.org; e-mail: ct@tbg.riogrande.com

\section{About the Author}

Mark Grimwade is an expert on precious metals and jewellery technology. A technical consultant to World Gold Council and the Goldsmiths' Company in London, Mark has published in Aurum and Gold Technology magazines in the series 'Basic Metallurgy for Goldsmiths' and reports on the Santa Fe Symposia. He is a frequent presenter at the Santa Fe Symposium of jewelry manufacturing technology. He wrote the book 'An Introduction to the Precious Metals', published in 1985 and the 'Handbook on Soldering', one of the Handbooks published in 2002 on jewellery technology by World Gold Council.

\section{Editors' note:}

These reviews have traditionally been published in the sister journal, Gold Technology, now no longer being published. We will publish a review of this year's Santa Fe Symposium in a later issue of Gold Bulletin. 